\title{
LAS CONDICIONES LABORALES Y HABITACIONALES DE LOS RECURSOS HUMANOS EN EL TAMBO. SANTA FE. ARGENTINA
}

\author{
Suero, M..$^{1}$ Brizi, M. C. ${ }^{2} \&$ DANIELE, M. ${ }^{3}$
}

\begin{abstract}
RESUMEN
El presente artículo analiza las condiciones laborales y habitacionales de los operarios tamberos de las empresas que integran la “Cooperativa Tambera Central Unida Limitada” de San Guillermo, provincia de Santa Fe. La importancia de este análisis radica en que los recursos humanos son fundamentales para que los sistemas de producción de leche sean competitivos. Para concretar dicha propuesta se realizaron entrevistas a productores/empresarios y a operarios/tamberos utilizando como metodología de investigación el estudio de casos. Los resultados obtenidos permitieron concluir que el estado habitacional de las viviendas, las condiciones laborales y los aspectos medioambientales influyen en el desempeño de los operarios. Además, el impacto de dichos factores depende de la escala productiva de la empresa.
\end{abstract}

Palabras clave: recursos humanos, operario tambero, condiciones laborales, condiciones habitacionales, empresa tambera.

\section{ABSTRACT}

\section{Working and housing conditions of human resources in a dairy farm in Santa Fe, Argentina.}

The present article analyzes the working and housing conditions of the dairy operators of the companies that integrate the "Central United Limited Dairy Cooperative” from San Guillermo, province of Santa Fe. The importance of this analysis lies in the fact that human resources are fundamental to ensure competitiveness of the milk production systems. To carry out this proposal, interviews were

1.- Grupo de Economía. Facultad de Ciencias Agrarias, Universidad Nacional del Litoral. Telefax 54-3496420439. E-mail: msuero@fca.unl.edu.ar. Kreder 2805. (3080) Esperanza, provincia de Santa Fe, Argentina. 2.- Grupo de Economía. Facultad de Ciencias Agrarias. Universidad Nacional del Litoral.

3.- Asesor privado.

Manuscrito recibido el 23 de octubre de 2019 y aceptado para su publicación el 25 de noviembre de 2019.

Suero, M.; Brizi, M. C.; Daniele, M. Las condiciones laborales y habitacionales de los recursos humanos en el tambo. Santa Fe. Argentina. FAVE - Ciencias Agrarias 18 (1): 73-82. CC BY-NC-SA 4.0 (c)(1)(2) 


\section{Suero et al.}

administered to producers and businessmen, and to dairy farmers and operators using the case study as the research methodology. The results obtained allowed us to conclude that the housing and working conditions, as well as the environmental aspects have a major influence upon the performance of operators. Besides, the impact of these factors depends on the company production scale

Key words: human resources, dairy operator, working conditions, housing conditions, dairy company.

\section{INTRODUCCIÓN}

Los cambios tecnológicos y de escala, junto a la intensificación de los procesos productivos de los sistemas lecheros, exigen que los recursos humanos en la empresa tambera tomen un rol importante para lograr la sustentabilidad de dichos sistemas en el tiempo. La lechería es una actividad muy dinámica, exigente y compleja en sus interrelaciones sociales, políticas, económicas y ambientales, lo que conlleva a que el personal se vea continuamente desafiado y deba incorporar nuevos conocimientos técnicos para lograr una mayor eficiencia y rentabilidad del sistema (3).

Gran parte del crecimiento y evolución de la actividad estuvo apoyada en la mayor utilización de insumos críticos, como la implementación de nuevas variedades forrajeras, y vacas de ordeño potencialmente más productivas, entre otros (10). Si bien estas tecnologías de insumos son fáciles de adquirir, las mismas por sí solas no garantizan la sostenibilidad del sistema productivo.

Lo difícil es la correcta implementación de las tecnologías de procesos como la rutina de ordeño, el lavado de la máquina, manejo de pasturas, manejo reproductivo, estacionamiento de las pariciones, crianza de terneros y la gestión de la actividad, todos ellos factores determinantes de la competitividad de la empresa. En dichas tecnologías de procesos intervienen los recursos humanos como prioridad, siendo significativa la gestión de los mismos dentro de la empresa.

Actualmente la provincia de Santa Fe es la segunda productora de leche del país. La misma produjo, en el período 2010-2013, el $32 \%$ de la leche a nivel nacional (13). Además, la Cuenca Lechera Central Santafecina concentra el $78 \%$ de los tambos y el $77 \%$ de las vacas a nivel provincial (6).

De esta manera cada tambo genera, en la provincia, entre dos y tres puestos de trabajo en forma directa, considerando que en la gran mayoría de los casos todos los miembros de la familia participan o colaboran con alguna tarea propia de la actividad (14). $\mathrm{Al}$ respecto, Osan, O., citado por Castignani et al. (2) verificó que la proporción de la mano de obra contratada aumenta con la escala de producción y, consecuentemente, se reduce el aporte de mano de obra familiar.

El recurso humano se percibe cada vez más crítico, pues requiere de mayor especialización y competencias; de hecho, la mala calidad del mismo suele operar como una limitante a la incorporación de tecnologías de procesos complejas (2).

Por otra parte, subsisten problemas para atraer y mantener mano de obra calificada a las empresas; algunos de ellos son derivados de la propia actividad productiva (horarios, y condiciones de trabajo), otros, son aspectos relacionados a la movilidad, comunica- 
ción, infraestructura (estado habitacional) y escolarización de los niños. En efecto, una encuesta realizada a tamberos de la Región CREA $^{1}$ Santa Fe Centro (2012) arrojó como resultado que el $53 \%$ de las personas que participaron como tamberos tenían menos de dos años en el puesto de trabajo, el 74\% veía al empleo como una actividad sacrificada y el 58\% no recomendaría esta actividad a sus hijos (13). Todo esto genera la dificultad de formar grupos de trabajos que perduren en el tiempo y que persigan los objetivos y las metas destinadas al crecimiento productivo y desarrollo de la empresa.

Por todo lo expuesto, puede afirmarse que el tambero es un actor clave en el funcionamiento de la empresa y más aún en aquellos establecimientos en los que, por su escala productiva, deben disponer de un mayor número de personal (tractoristas y peones) para poder realizar todas las tareas que hacen a la actividad en su conjunto. El objetivo principal del trabajo es conocer las condiciones laborales y habitacionales de los recursos humanos en el tambo, como así también caracterizar el tipo de empresas a donde trabajan y cómo están conformados los grupos de trabajo.

\section{MATERIALES Y MÉTODOS}

El trabajo de investigación se desarrolló en los sistemas productivos primarios de leche pertenecientes a la "Cooperativa Tambera Central Unida Limitada” (Asociada a SanCor Cooperativas Unidas Limitada $N^{\circ}$ 484), que cuenta con 96 tambos, ubicada en San Guillermo, departamento San Cristóbal, provincia de Santa Fe. La misma integra la
“Asociación de Clúster Lechero Regional”: Asociación para el Desarrollo Regional de la Cadena Láctea del Noroeste de Santa Fe y el Sureste de Santiago del Estero (C.L.E.R.).

Como metodología de investigación científica se utilizó el método de estudio de casos. Los datos pueden ser obtenidos desde una variedad de fuentes como: documentos, entrevistas directas, observaciones directas o de los mismos participantes (Chetty, citado por Carazo, M. \& Piedad, C.) (4).

En lo que refiere al análisis, se consideró el período junio 2016 a julio 2017. Cabe aclarar que en este ciclo productivo se produjo el evento meteorológico "El Niño" de gran intensidad e impacto sobre la zona de influencia.

Antes de comenzar con el trabajo de campo se realizó un análisis de la distribución poblacional de los tambos y una estratificación por producción diaria de leche entregada (5). Se conformaron tres categorías o grupos. El grupo A, corresponde a tambos cuyo volumen de entrega de leche diaria no supera los 1000 l/día; el grupo B de los 1001 1/día hasta los 2500 l/día y el grupo C comprende a aquellos tambos con una producción de leche mayor a los 2500 l/día.

Bajo la metodología de estudio de casos no hay un número preciso de entrevistados a ser estudiados en cada grupo, se deben ir incorporando casos hasta que la saturación teórica sea enriquecida (4) o lo que otros autores denominan punto de la redundancia. Se seleccionaron en total nueve casos, tres por grupo, y se realizaron entrevistas personales, mediante un cuestionario semi estructurado, a los productores/empresarios y a los operarios del tambo; obteniéndose en total dieciocho entrevistas.

1.- Grupos CREA o AACREA: Asociación Argentina de Consorcios Regionales de Experimentación Agrícola. 
Luego del trabajo de campo se procedió a organizar y procesar los datos obtenidos, los cuales se volcaron a una hoja de cálculo para sistematizar la información y así poder analizarlos.

\section{ANÁLISIS DE LOS RESULTADOS}

\section{Características de las empresas tamberas}

Las empresas que integran el Grupo A, presentan una organización simple, conformada por un empresario individual; operan una superficie no mayor a las 250 hectáreas (ha), destinadas en su totalidad a la producción de leche, como principal actividad y fuente de ingreso. El plantel de vacas en ordeño no supera los 100 animales manejados en un solo rodeo. Las instalaciones de ordeño son diversas.

Las empresas del Grupo B, son más complejas respecto a su estructura y actividad. Dicho grupo opera una superficie promedio de 350 ha destinadas a la actividad tambera en el que completan todo el ciclo de producción. Cuentan con un plantel de vacas en ordeño de alrededor de 120 animales, divididos en rodeos de punta y cola. Poseen instalaciones en sus tambos con fosa tipo espina de pescado y la mayoría tiene corrales de espera de hormigón.

En las empresas que conforman el Grupo C la actividad tambera no es la única fuente de ingresos económicos, pero sí la más importante, existiendo un mayor grado de especialización. La superficie operada fluctúa entre las 300 y 800 ha, destinadas a la producción lechera, agrícola y ganadera. El rodeo lechero oscila entre las 180 a 350 vacas en ordeño, divididos en lotes, de punta y cola. Todos los tambos poseen instalaciones de ordeño con fosas de tipo espina de pescado y corrales con de piso de cemento y sombra artificial.

Existen diferencias entre los tres grupos respecto a las producciones individuales de las vacas en ordeño. El grupo C tiene una producción individual promedio de $23 \mathrm{l}$ de leche vaca/día, el grupo B, 19 l de leche vaca/día, es decir 17\% menor que el anterior, mientras que en el grupo A es de $13 \mathrm{l}$ de leche vaca/día. Es decir que el grupo A produce un $45 \%$ menos que el grupo $\mathrm{C}$ y un 33\% menos que el Grupo B. La disparidad que se manifiesta, principalmente entre los dos grupos extremos, puede explicarse en parte, al manejo productivo que realizan estos; en el grupo A no existe división de rodeos según su estado productivo, la utilización de concentrados es a base de maíz y los forrajes conservados sólo aportan volumen y no son de buena calidad. La utilización de inseminación artificial es inexistente. En el grupo $\mathrm{C}$ el rodeo lechero se encuentra dividido según su estado productivo pudiendo de esta manera aportar cantidad y calidad de alimentos para cubrir las demandas fisiológicas y obtener altos valores productivos, la utilización de concentrados junto a alimentos de excelente calidad y cantidad, permite formar dietas totalmente balanceadas según estas necesidades. La inseminación artificial es una herramienta que permite mejorar la calidad genética del rodeo en lo que respecta a producción y calidad de leche.

Todos los grupos cuentan con superficie arrendada en porcentajes variables.

\section{Características de los operarios tamberos de las empresas lecheras}

Los grupos de trabajo son variados, el número de integrantes de los mismos depende de la escala productiva y de las actividades que deban desarrollar en la empre- 
sa. Las familias de los tamberos se ajustan a la denominada "familia tipo", formadas por cuatro personas que pertenecen al núcleo familiar encontrándose grupos formados por 3 y 5 miembros.

Se identificaron 27 operarios tamberos, entre todos los casos, que participan dentro de la empresa realizando diferentes actividades, con un rango de edad entre los $18 \mathrm{y}$ 45 años.

Respecto al nivel educativo, es importante destacar el alto grado de escolaridad incompleta que se encuentra entre los operarios, siendo un porcentaje muy bajo el que cuenta con nivel secundario completo. Manifestaron como motivos de la deserción escolar, la temprana edad de incorporación al ámbito laboral, dificultades en el traslado a colegios de zonas urbanas para realizar el nivel secundario y la falta de incentivos por parte de sus padres. Sin embargo, el trabajo arrojó datos importantes que muestran una tendencia de reversión de esta situación. El $40 \%$ de los entrevistados exteriorizaron la importancia del estudio y el deseo de que sus hijos finalicen el mismo antes de ingresar al ámbito laboral. Estos datos no se alejan de los que refleja el grupo CREA de la Región Santa Fe Centro, que bajo una población de 302 empleados de tambos (entre ellos ordeñadores, tamberos, vaqueros, encargados, tractoristas y guacheros) encontró que el $69 \%$ de los encuestados poseían solo estudios primarios, el 8\% estudios secundarios y el 2\% universitarios (9), mientras que Sandoval et al. (11) en un estudio realizado sobre la misma zona de influencia que el caso anterior, encontró que las escuelas primarias rurales están perdiendo progresivamente las matrículas.

En lo que respecta a las actividades laborales cotidianas, la participación de la mujer es muy activa, logrando un equilibrio entre las labores hogareñas y las correspondientes al trabajo. Las mismas intervienen en actividades como: rutina de ordeño, detección de celos y servicios, atención a partos, tratamientos veterinarios, siendo exclusivo en la mayoría de los casos la crianza de terneros. Los hombres realizan las actividades nombradas anteriormente incluyendo el manejo de pasturas (boyeros eléctricos) y actividades extras como el suministro de forrajes conservados, desmalezado, mantenimiento de callejones y corrales, entre otros.

\section{Condiciones y medio ambiente de trabajo del operario tambero}

Se considera como medio ambiente de trabajo a todos los "elementos reales que inciden directa o indirectamente en la salud de los trabajadores; constituyen un conjunto que obra en la realidad concreta de la situación laboral”. El Programa Internacional para el mejoramiento de las condiciones y Medio Ambiente de trabajo (PIACT) establece entre sus principios: a) el trabajo debería realizarse en un ambiente seguro y salubre; b) las condiciones de trabajo deberían ser compatibles con el bienestar y la dignidad humana y c) el trabajo debería ofrecer al trabajador posibilidades reales de desarrollar su personalidad y de servir a la sociedad (9).

\section{Estado habitacional y disponibilidad de servicios}

La vivienda es una necesidad humana fundamental, al igual que la alimentación y el vestido, su carencia priva al ser humano de lograr una mejor calidad de vida (8).

Las viviendas de los casos entrevistados son muy diversas entre sí, su tamaño y diseño depende del número de personas 
o familias que las habitan. Generalmente son casas con varios años de construcción y remodeladas según las necesidades habitacionales. Se puede describir una casa tipo formado por una cocina comedor, dos habitaciones y un baño, paredes de mampostería de ladrillo asentada en barro o cemento (dependiendo de la antigüedad), todas con revoque fino pintado, los techos son de chapa de zinc con cielo raso de madera o PVC. En el caso de poseer techos de material estos fueron recubiertos en su totalidad por chapa o membrana plástica ya que poseen filtraciones, los pisos son de losa de cemento recubiertos por cerámicos, baldosas antiguas o pintadas.

Respecto al circuito de agua por cañería, el mismo es variable, todos los evaluados poseían agua por cañería, tanto fría como caliente en el baño, pero no así en el lavadero y cocina.

La energía eléctrica llega a los hogares por medio de la red eléctrica rural, y el suministro es continuo. En el caso de eventuales cortes existen generadores eléctricos para ser accionado con la toma de fuerza de un tractor o motor a explosión independiente, que sólo proporcionan electricidad al tambo y son utilizados, únicamente, durante el ordeño y para el enfriado de la leche. Al momento de calificar la vivienda según su estado habitacional, todos los operarios coincidieron que las mismas se encontraban en un estado entre "bueno y muy bueno".

Las viviendas correspondientes al grupo A se caracterizan por poseer estructuras antiguas ausentes de mantenimiento, con ambientes grandes, techos con loza de cemento o chapa de zinc todos con cobertura de membrana plástica. Las paredes exteriores pueden estar o no revocadas mientras que las interiores se encuentran pintadas. Los pisos son de losa de cemento recubiertos por mosaicos antiguos, dos casos poseían circuito de agua fría en cocina y fría y caliente en el baño. El otro caso no cuenta con agua por cañería en la casa y el baño con instalación completa de sanitarios se encuentra en el tambo. Las conexiones eléctricas manifiestan deficiencias y hasta poseen reparaciones y conexiones "caseras". En cuanto al grupo B, las viviendas, en lo que concierne a antigüedad y estructura, no difieren respecto al grupo anterior pero sí lo hacen en su estado general (mejor estado de mantenimiento) y equipamiento. Todas poseen agua por cañería en la cocina, los baños son internos, con equipamiento completo y circuito de agua fría y caliente y el mismo se utiliza como lavadero. Dos viviendas poseen techos de chapa de zinc con cielo raso mientras que el otro es de cemento.

Finalmente, el grupo $\mathrm{C}$ es el que mejor estado habitacional presenta, existen casas de construcciones antiguas y modernas, todas están en muy buen estado de mantenimiento, los techos son predominantemente de chapa de zinc con cielo raso de madera o PVC, las que poseen loza de cemento están recubiertas por membrana plástica. Las paredes internas y externas se encuentran es su totalidad pintadas, las conexiones eléctricas están en muy buen estado, pudiendo ser externas o empotradas dentro de la pared. Las externas cuentan con cable-canal. La cocina cuenta con equipamiento completo, con agua fría y caliente, baños internos completos provistos de agua fría y caliente. Una de las viviendas posee servicio de televisión satelital y Wifi abonado por el propietario del establecimiento, además en casos de corte de luz poseen un motor a explosión independiente que brinda electricidad tanto al tambo como a la casa. 
Durante la entrevista, dos productores del presente grupo, manifestaron el alto grado de importancia que le otorgan ellos a la comodidad habitacional de los operarios. Al visitar los establecimientos se pudo constatar lo dicho, a su vez uno de estos expresó que en el contrato de trabajo que posee con el tambero, se detalla el estado con que se entrega la casa cuando éste ingresa a la empresa y en el que debe devolverse.

Seis de los casos poseían más de una familia viviendo en el mismo establecimiento, estas familias son peones de tamberos o tractoristas, todos poseen unidad habitacional individual. En tres casos, la unidad habitacional consistía en una casa grande que fue reformada para constituir dos unidades habitacionales.

Instalaciones de ordeño (lugar de trabajo) y jornada laboral

El adecuado dimensionamiento de las instalaciones de ordeño es esencial para realizar el trabajo de forma eficiente. Cada equipo, no debería realizar las tareas de ordeño por un tiempo que supere las dos horas consecutivas (1). De esta forma se maximizan las posibilidades de lograr procesos eficientes y mantiene la motivación de las personas que trabajan en el tambo. La carencia de infraestructura genera mayor tiempo de operación, mayor esfuerzo y consecuentemente, ineficiencias productivas y peores condiciones laborales.

Entre los casos entrevistados se han observado diversas estructuras destinadas a la extracción de leche. Las mismas varían en tamaño y diseño dependiendo principal- mente de los materiales de construcción, reformas, antigüedad y del número de animales destinados al ordeño. Siete casos poseen salas de ordeño tipo espina de pescado, con equipo de ordeño simple (hay una unidad de ordeño compartida entre ambos lados de la fosa) y línea de leche superior, mientras que dos poseen bretes a la par, uno con paso a través y otro con retrocesos. El número de bajadas $^{2}$ depende generalmente del tamaño del rodeo lechero, aunque es notorio, que éste no siempre condice con el número de animales. Ente los casos analizados se destacan las instalaciones subdimensionadas, es decir poseen una relación $\mathrm{VO}^{3} / \mathrm{N}^{0}$ de bajadas, menor a lo recomendado (15 VO/ bajada) (1). Esta situación es más frecuente en aquellos tambos de elevada producción y números de animales (los pertenecientes al grupo B y C). Los resultados, en los tambos del grupo C, arrojaron un promedio de 19 VO/bajada, es decir un 27\% superior a lo aconsejado, $16 \mathrm{VO} /$ bajada en el grupo $\mathrm{B}$, esto es un $7 \%$ superior y $12 \mathrm{VO} /$ bajada, es decir un 20\% debajo de lo requerido, en el grupo A. En promedio, cada operario atiende por ordeño $36 \mathrm{VO}$ en el grupo A, $51 \mathrm{VO}$ en el grupo B y 74 vacas en el grupo C.

El dimensionamiento inadecuado de las instalaciones de ordeño aumenta la jornada de trabajo afectando tanto al personal a cargo de la tarea como a los animales. Así, a las vacas les genera estrés al tener que esperar un tiempo prolongado para ser ordeñadas, aumentando la cantidad de leche retenida y repercutiendo tanto en la producción individual como en la salud de la ubre. Con respecto al operario, el aumento de la jor-

2.- Bajadas o unidad de ordeño: compuesta por las pezoneras, el colector de leche, tubo largo de leche, tubo largo de pulsación y un pulsador.

3.- VO: Vaca en ordeño o lactancia. 
nada laboral produce cansancio tanto físico como mental lo que impacta en la rutina de ordeñe $^{4}$ y se encuentra propenso a cometer errores, desatenciones y provocar accidentes. Es notoria la diferencia que existe en la duración de la rutina de ordeño entre los dos grupos extremos, mientas que en el grupo $\mathrm{A}$ la duración es, en promedio, de 2 horas por ordeño, la del grupo $C$ se extiende unas 3,5 horas. Esta diferencia puede explicarse por lo tratado en el párrafo anterior, los tambos del grupo A presentan una capacidad ociosa de trabajo, es decir, admiten mayor número de vacas que las que actualmente se encuentran en ordeño (ocupan un $80 \%$ de la capacidad) por lo que la rutina se acorta. En los tambos del grupo C su capacidad operativa se encuentra sobredimensionada.

En todos los casos analizados, a la rutina de ordeño se anexan trabajos como la detección de celos e inseminación artificial (en algunos casos), atención de partos, manejo de la guachera, suministro de pasturas y tratamientos veterinarios, que demandan mayor o menor cantidad de horas de trabajo, según su complejidad y cantidad de personas que se ocupan de las mismas. En los tambos del grupo $\mathrm{A}$, a las actividades mencionadas anteriormente hay que adicionarles otras complementarias como el suministro de forrajes conservados, desmalezado de pasturas y mantenimiento de las instalaciones en general. Castignani et al. (2), destaca que en las empresas con mano de obra contratada y bajo volumen de producción diaria, los operarios tienen bajo su responsabilidad un mayor número de actividades, esta situación genera sobre ellos un mayor grado de malestar, sobre todo si las remuneraciones no son las adecuadas respecto a las actividades que realizan. En los establecimientos de alta producción, se observa mayor especialización en las actividades, pero a su vez, mayores conflictos interpersonales.

\section{CONCLUSIONES}

Se pudo observar, mediante los casos analizados, que el estado habitacional de las viviendas, las condiciones laborales y los aspectos medioambientales influyen en el desempeño de los operarios tamberos.

Así mismo se observó que al aumentar la escala de producción, la estructura organizativa se torna más compleja y se incrementa el nivel de integración de las actividades en la empresa. Por consiguiente, los grupos de trabajos se encuentran conformados por un mayor número de integrantes siendo necesaria la contratación de mano de obra no familiar. Por otro lado, a medida que la productividad y la escala aumentan, la tecnología aplicada al sistema de producción demanda más subdivisión y especialización de las tareas. Por lo tanto, en estos casos, se torna significativo y sumamente importante gestionar de manera eficiente los recursos humanos de la empresa.

En último término se puede decir que el recurso humano es el capital más valioso que poseen las empresas tamberas, por lo tanto, es fundamental que el productor/empresario comprenda la importancia del mismo y asegure las buenas condiciones de trabajo y habitacionales para que el sistema de producción lechera sea realmente competitivo.

4.- Rutina de ordeño: tiempo que trascurre desde el ingreso de los animales al tambo hasta finalizar con la limpieza de las instalaciones. 


\section{AGRADECIMIENTOS}

A la Cooperativa Tambera Central Unida Limitada” de San Guillermo, por permitir realizar esta investigación, a los productores y operarios por su predisposición para responder a las entrevistas y a todos los profesionales involucrados.

\section{BIBLIOGRAFÍA}

1.- BAUDRACCO, J.; LAZZARINI, B.; BREGA, M.; BOUMAN, METTE.; LYONS, N. Y CUADRADO, C. 2018. Instalaciones de ordeño para agilizar y simplificar el trabajo: Pensando en una nueva instalación de ordeño. Proyecto Interinstitucional Factor Humano en Tambo Argentino. Disponible en: http:// factorhumanoentambo.com/equipamiento-para-simplificar-procesos/Acceso: 26/09/2019

2.- CASTIGNANI, M. I.; CURSACK, A.M.; BLANGETTI, E.; OSAN, O. Y ROSSLER, N. 2009. Los recursos humanos en la empresa lechera: un análisis de su relación con el perfil tecnológico y estructural mediante estudios de casos. VIII Jornada Interdisciplinarias de Estudios Agrarios y Agroindustriales. Centro Interdisciplinarios de Estudios Agrarios. Fac. de Cs. Económicas, UBA. Buenos Aires. Trabajo completo. 19 p.Disponible en: http://www. fca.unl.edu.ar/tictambo/web/docs/RecHumanosEmpresaLechera.pdf

3.- CASTIGNANI, M.I.; OSAN, O.; ROSSLER, N.; MAINA, M.; BRIZI, M. C. Y ACOSTA, L. 2013. Herramientas para la gestión del capital humano en empresas tamberas de la Cuenca Central Santafecina. VIII Jornada Interdisciplinarias de Estudios Agrarios y Agroindustriales. Centro Interdisciplinarios de Estudios Agrarios. Fac. de Cs. Económicas, UBA. Buenos Aires. Trabajo completo. 19 p.
4.- CARAZO, M. Y PIEDAD, C. 2006. El método de estudio de casos: estrategia metodológica de la investigación científica. Pensamiento \& Gestión 20. Universidad del Norte Barranquilla, Colombia. Pp. 165 - 193. ISSN 1657-6276.

5.- COOPERATIVA TAMBERA CENTRAL UNIDA LTDA. 2016. Estadísticas de Producción. [online] Disponible en: http://www.ccunida.com.ar/index.php/features/2015-06-01-09-45-26

6.- CURSACK, A. M.; TRAVADERO, M.; CASTIGNANI, M. I.; OSAN, O.; SUERO, M.; CASTIGNANI, O.; MAINA, M. Y BRIZI, M.C. 2006. El sector lechero en la Cuenca Central Santafesina. Investigaciones sobre los factores condicionantes de la competitividad microeconómica. I Jornada: La vinculación de las investigaciones de la Universidad Nacional del Litoral con el sector lácteo de la Región Centro. Fac. de Cs. Económicas, UNL. Santa Fe. 24p. [online] Disponible en:http://www.fca.unl.edu.ar/tictambo/web/ docs/InvestigacionesCondicionantesCompetitividad.pdf

7.- MARCHISIO, C. 2013. Encuesta: El tambo según sus recursos humanos. Grupos CREA de la Región Santa Fe Centro. En: http:// www.infortambo.com/ Acceso: 19/09/2019.

8.- MAX NEEF, M.; ELIZALDE, A. Y HOPENHAYN, M. 1993. Desarrollo a Escala Humana. Conceptos, aplicaciones y algunas reflexiones. Icaria Editorial. Montevideo, Uruguay. $152 \mathrm{p}$.

9.- NICOLACI, M. 2008. Condiciones y medio ambiente de Trabajo (CyMAT). Hologramática. Revista Académica de la Facultad de Ciencias Sociales UNLZ 8 (2), pp.3-48. ISSN 1668-5024 Disponible en: http://www. cienciared.com.ar/ra/doc.php?n=835

10.- OSAN, O. 2005. Dirección de empresas: hablemos de los procesos. Revista SanCor 657: 28-31. 
11.- SANDOVAL, P.; LEONARDI, R.; PER- 13.- TAVERNA, M. Y FARIÑA, S. 2013. SisNUZZI, C.; MARTINS, L.; BENÍTEZ, R.; ARNAUDO, J.; EGGEL, A.; BRANCE BONVINI, M. I.; ACOSTA, G.; ZUSKA, G. Y RUFINO, E. 2016. ¿Cuáles son las necesidades de los tamberos actuales, en la cuenca lechera santafesina? Un estudio de caso en la zona lechera central, Santa Fe, Argentina. XVIII Jornadas Nacionales de Extensión Rural y X del Mercosur: "La Extensión Rural y los modelos de Desarrollo en el año del bicentenario”. Fac. de Cs. Agrarias. UNCo. Río Negro. 6 p.

12.- TAVERNA, M. Y FARIÑA, S. 2013. La Producción de Leche en Argentina; p.730. En: Campbell, J.A. (ed.). Anuario de la Lechería Argentina 2013. Fundación para la Promoción y el Desarrollo de la Cadena Láctea Argentina. Inforcampo S.A. Vicente López, Buenos Aires. tema de Producción de Leche en Argentina. Su caracterización; p. 19-23. En: Campbell, J.(ed.). Anuario de la Lechería Argentina 2013. Fundación para la Promoción y el Desarrollo de la Cadena Láctea Argentina. Inforcampo S.A. Vicente López, Buenos Aires. Argentina.

14.- TERÁN, J. C. 2008. Caracterización de la cadena agroalimentaria de la leche en la provincia de Santa Fe. INTA. Rafaela. 21 p. 